\title{
Transition from Restrictive to Obstructive Lung Function Impairment During Treatment and Follow-Up of Active Tuberculosis
}

This article was published in the following Dove Press journal: International Journal of Chronic Obstructive Pulmonary Disease

\author{
Brian W Allwood (D) ${ }^{1,2}$ \\ Elizna Maasdorp (iD) ${ }^{3}$ \\ Grace J Kim ${ }^{4,5}$ \\ Christopher B Cooper $\mathbb{D}^{6}$ \\ Jonathan Goldin ${ }^{4}$ \\ Richard N van Zyl-Smit (iD) ${ }^{2}$ \\ Eric D Bateman ${ }^{2}$ \\ Rodney Dawson ${ }^{2}$
}

'Division of Pulmonology, Department of Medicine, Stellenbosch University, Cape

Town, South Africa; ${ }^{2}$ University of Cape

Town Lung Institute, and Division of Pulmonology, Department of Medicine, University of Cape Town, Cape Town, South Africa; ${ }^{3}$ DST/NRF Centre of Excellence for Biomedical Tuberculosis Research; South African Medical Research Council Centre for Tuberculosis Research; Division of Molecular Biology and Human Genetics, Faculty of Medicine and Health Sciences, Stellenbosch University, Cape Town, South Africa; ${ }^{4}$ Center for Computer Visions and Imaging Biomarkers, Department of Radiology, David Geffen School of Medicine, University of California, Los Angeles, CA, USA;

${ }^{5}$ Department of Biostatistics, Fielding School of Public Health, University of California, Los Angeles, CA, USA;

${ }^{6}$ Departments of Medicine and Physiology, David Geffen School of Medicine, University of California, Los Angeles, CA, USA

Correspondence: Brian W Allwood Division of Pulmonology, Department of Medicine, Stellenbosch University, Rm 3013 3rd Floor; Clinical Building; Francie Van Zijl Drive; Medical School, Tygerberg 7505, South Africa

Email brianallwood@gmail.com
Background: Pulmonary tuberculosis (PTB) is associated with many forms of chronic lung disease including the development of chronic airflow obstruction (AFO). However, the nature, evolution and mechanisms responsible for the AFO after PTB are poorly understood. The aim of this study was to examine the progression of changes in lung physiology in patients treated for PTB.

Methods: Immunocompetent, previously healthy, adult patients receiving ambulatory treatment for a first episode of tuberculosis were prospectively followed up with serial lung physiology and quantitative computed tomography (CT) lung scans performed at diagnosis of tuberculosis, 2, 6, 12 and 18 months during and after the completion of treatment.

Results: Forty-nine patients (median age 26 years; 37.2\% males) were included, and 43 were studied. During treatment, lung volumes improved and CT fibrosis scores decreased, but features of AFO and gas trapping emerged, while reduced diffusing capacity (DLco) seen in a majority of patients persisted. Significant increases in total lung capacity (TLC) by plethysmography were seen in the year following treatment completion (median change $5.9 \%$ pred., $\mathrm{P}<0.01$ ) and were driven by large increases in residual volume (RV) (median change $+19 \%$ pred., $\mathrm{P}<0.01$ ) but not inspiratory capacity (IC; $\mathrm{P}=0.41$ ). The change in $\mathrm{RV} /$ TLC correlated with significant progression of radiological gas trapping after treatment $(\mathrm{P}=0.04)$ but not with emphysema scores. One year after completing treatment, $18.6 \%$ of patients had residual restriction (total lung capacity, TLC $<80 \%$ pred), $16.3 \%$ had AFO, $32.6 \%$ had gas trapping (RV/TLC $>45 \%$ ), and $78.6 \%$ had reduced DLco.

Conclusion: Simple spirometry alone does not fully reveal the residual respiratory impairments resulting after a first episode of PTB. Changes in physiology evolve after treatment completion, and these findings when taken together, suggest emergence of gas trapping after treatment likely caused by progression of small airway pathology during the healing process.

Keywords: tuberculosis, post-tuberculosis, chronic obstructive pulmonary disease, lung function, computed tomography, airflow obstruction

\section{Plain Language Summary}

Pulmonary tuberculosis is known to cause permanent damage to the lungs in some individuals. This study shows that in previously healthy, young (median age of 26 years), immunocompetent adults, routine spirometry underestimates the amount of residual damage after a first episode of tuberculosis, when more specialized physiology tests are also used to assess patients. More importantly, it shows that changes to the lung occur even after treatment completion. In the year after finishing treatment, there were significant increases in lung volumes, and these increases were largely due to increases in residual volume (the 
volume remaining in the lung after full expiration). This physiological change implies that after treatment completion gas trapping may emerge. This was confirmed on CT scanning of the lung in a subset of patients where physiological changes correlated significantly with imaging changes of gas trapping. When taken together, this physiological-radiological comparison suggests that after tuberculosis, changes may occur to the small airways, likely narrowing, resulting in the development of gas trapping and airflow obstruction.

\section{Introduction}

Pulmonary tuberculosis (PTB) may cause permanent impairment of lung function and is associated with several forms of chronic lung disease. ${ }^{1-8}$ An association between past PTB and chronic airflow obstruction (AFO) has been reported in large population-based epidemiological studies ${ }^{3-6}$ and contributes to the heavy burden of chronic airways disease seen in high PTB prevalence communities. A causal relationship is suggested by studies examining changes in lung function in patients completing PTB. ${ }^{7,9-13}$ There is, however, little published evidence for the mechanisms responsible for the impairment of lung function and its clinical course. A common assumption is that the more striking and characteristic sequelae of PTB, namely bronchiectasis, fibrosis, and bronchial stenosis are the major mechanisms responsible for $\mathrm{AFO},{ }^{2,3,14}$ but few studies have attempted a more thorough assessment of regional structure-function relationships within affected lungs. Nor has it been established whether lung function impairment progresses after the active TB infection has been successfully treated. In a previous observational study of patients with a past history of PTB and spirometric evidence of AFO, we have confirmed that static gas trapping and reduced diffusing capacity (DLco) are common findings, and we have hypothesized that this is best explained by widespread cicatricial changes in or surrounding bronchovascular bundles containing small airways. ${ }^{15}$ Further, this process may progress over time and contribute to chronic airflow obstruction seen later after treatment has been completed. This observation raises the possibility of considering interventions that might limit these late effects of PTB.

We report here the results of a prospective study of the serial changes in lung function and quantitative CT findings in a cohort of patients with active PTB treated for six months and followed for one year after completion of treatment for PTB, and describe the nature and course of the pulmonary sequelae of PTB during and after completion of treatment.

\section{Materials and Methods Subjects}

Patients with newly diagnosed drug-sensitive pulmonary TB were recruited from two local ambulatory TB clinics. Eligible patients gave written consent, were 18 years or older with active PTB diagnosed on sputum nucleic acidamplification testing, confirmed by microscopy and culture. Patients were required to be HIV negative or HIV positive with a CD4 count of over 250 cell $/ \mu L$. Exclusion criteria were a history of previous PTB, known chronic lung disease or malignancy; a history of illicit drug use; pregnant at the time of diagnosis; or a diagnosis of drug-resistant tuberculosis. Consecutive patients meeting enrollment criteria on recruitment days were invited to participate, with a maximum of three patients recruited per day. For inclusion in the analysis, patients were required to complete an outpatient course of standard anti-tuberculosis therapy (6 months).

\section{Study Protocol}

Enrolled patients attended for five study visits: On the day of PTB diagnosis (Visit 1), and at 2, 6, 12 and 18 months thereafter (Visits 2 to 5 , respectively). At each visit, subjects performed a six-minute walk test, and spirometry before and after administration of an inhaled bronchodilator salbutamol 200ug from a pressurized metered-dose inhaler (pMDI), according to ATS/ERS standards, and ${ }^{16}$ Global Lung Initiative (GLI) reference equations were used. Body plethysmography and diffusing capacity for carbon monoxide (DLco) corrected for hemoglobin, were performed at 2, 6 and 18 months, and the St. George's Respiratory Questionnaire (SGRQ) at 6 and 18 months. Plethysmography and DLco were not performed prior to 2 months to reduce the risk of infection to staff.

Chronic obstructive pulmonary disease is currently defined by the presence of airflow obstruction identified by having FEV1/FVC $<0.70,{ }^{17}$ however, this may underestimate disease in younger persons. Airflow obstruction was defined as a post-bronchodilator ratio of forced expiratory volume in $1 \mathrm{sec}\left(\mathrm{FEV}_{1}\right)$ to forced vital capacity (FVC) (ie, $\mathrm{FEV}_{1} / \mathrm{FVC}$ ) of less than the predicted lower limit of normal (LLN). Restriction was defined as total lung capacity (TLC) less than $80 \%$ predicted, and gas trapping on plethysmography was defined as a residual 
volume (RV) to TLC ratio (RV/TLC) of greater than $45 \%$. A low DLco (corrected for hemoglobin) and DLco with alveolar volume (VA) correction (ie, DLco/VA) were defined as values of less than $80 \%$ predicted.

All patients underwent three high-resolution computed tomography (HRCT) scans of the chest, using a low-dose, non-contrasted protocol that included both inspiratory and expiratory scans. Patients were randomized (1:1:2) into three groups. Group 1 had HRCT scans at Visit 1 (baseline) and after 2 weeks and one month of treatment, Group 2 had scans at baseline, 1 and 2 months, and Group 3 had scans at 2 (Visit 2), 6 (Visit 3) and 18 months (Visit 5).

CT scan images were analyzed quantitatively using inhouse software (Los Angeles, USA). ${ }^{18}$ Using inspiratory scans (ie, TLC scans), percentages of low-attenuation areas (LAAs) $<-950 \mathrm{HU}$ were used to define emphysema, and fibrosis was measured as the quantity of lung fibrosis (QLF) of high-attenuation areas (HAAs) $>200 \mathrm{HU}$, either as a percentage (QLF (\%)) or millilitre volume (QLF $(\mathrm{mL})$ ). On the expiratory scans (ie, RV scans), the percentage of LAAs $<-856 \mathrm{HU}$ on expiratory (RV) scans was used to calculate the gas-trapping score (GTS).

This study was conducted in accordance with the Declaration of Helsinki, patient consent was written informed consent and ethical approval for the study was obtained from the University of Cape Town Human Ethics Committee (REF: 248/2013).

Statistical analysis was performed in the statistical programming language $\mathrm{R}$ version 3.4.1 (www.r-project. org/) and STATA version 12 (StataCorp, Texas, USA). Descriptive statistics are reported as means and standard deviations when variables are approximately normally distributed and as medians and interquartile ranges when not. Testing for significant differences was done using the Wilcoxon Signed-Rank test for paired data that was not normally distributed. For the difference testing, no correction for multiplicity was applied (38 comparisons were made). Simple linear regression was used for correlations between lung function and radiological variables. Sensitivity analysis for the influence of outliers on the regression was performed, and alpha for all tests was set at 0.05 .

\section{Results}

A total of 49 patients were recruited. Six patients were excluded from the long-term outcome analysis (see Figure 1). For the remaining 43 subjects, 16 (37.2\%) were male, median age of 26 years and 31 (72.1\%) were aged 40 years or less. Baseline characteristics and smoking status are reported in Table 1. The mean time to Visits 2, 3 and Visit 5 were 8.1 weeks, 26.3 weeks and 81.1 weeks, respectively. Four subjects delayed Visit 5 due to pregnancy (all conceived after treatment completion).

\section{Physiological Changes Over Time (Table 2)} Between baseline and 6 months values for $\mathrm{FEV}_{1}, \mathrm{FVC}$ but not for $\mathrm{FEV}_{1} / \mathrm{FVC}$ increased. The majority of the improvements occurred in the first two months of treatment for both $\mathrm{FEV}_{1}$ (median change $=4.6 \%$ pred., $\mathrm{P}<0.01$ ) and FVC (median change $=4.5 \%$ pred., $\mathrm{P}<0.01$ ). Smaller, but statistically significant changes were also observed between 2 and 6 months for $\mathrm{FEV}_{1}$ (median change = $2.9 \%$ pred., $\mathrm{P}<0.01$ ) and FVC (median change $=4.9 \%$ pred., $\mathrm{P}<0.01)$, but not for $\mathrm{FEV}_{1} / \mathrm{FVC}(\mathrm{P}=0.39)$. Between

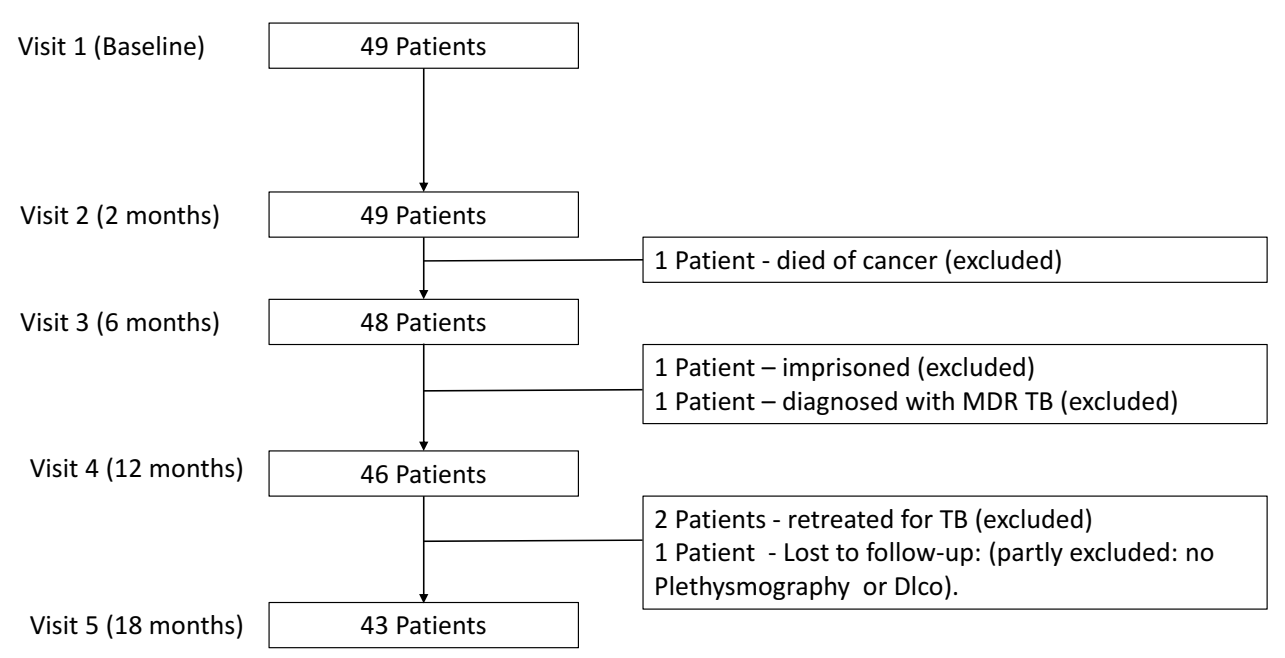

Figure I Recruitment and exclusion of subjects. 
Table I Baseline Demographic and Spirometric Characteristics

\begin{tabular}{|c|c|}
\hline Population Characteristics & $\begin{array}{l}\text { Number }(\% \text { of } \\
\text { Patients) }\end{array}$ \\
\hline \multicolumn{2}{|l|}{$(n=43)$} \\
\hline Male - n (\%) & $16(37.2 \%)$ \\
\hline Age - years, median (range) & 26 (20 to 65$)$ \\
\hline \multicolumn{2}{|l|}{ Race } \\
\hline African - n (\%) & $10(23.3 \%)$ \\
\hline Mixed race - n (\%) & $33(76.7 \%)$ \\
\hline \multicolumn{2}{|l|}{ Smoking Status } \\
\hline Never smokers - n (\%) & $12(27.9 \%)$ \\
\hline Former-smokers - n (\%) & II (25.6\%) \\
\hline Current smokers - n (\%) & $20(46.5 \%)$ \\
\hline $\begin{array}{l}\text { Pack years for ever smokers - median } \\
\text { (IQR) }\end{array}$ & $3.0(1.4-8.1)$ \\
\hline HIV positive - n (\%) & $2(4.7 \%)$ \\
\hline \multicolumn{2}{|l|}{ Baseline Spirometry (Post-Bd) } \\
\hline & Median (IQR) \\
\hline $\mathrm{FEV}_{1}(\mathrm{~L})$ & 1.97 (1.50 to 2.76$)$ \\
\hline FEV (\%pred.) & 0.59 (0.50 to 0.77$)$ \\
\hline $\mathrm{FVC}(\mathrm{L})$ & 2.49 (1.90 to 3.02$)$ \\
\hline FVC (\%pred.) & 0.63 (0.54 to 0.73$)$ \\
\hline $\mathrm{FEV}_{\mathrm{l}} / \mathrm{FVC}(\%)$ & $81.9(75.2$ to 88.1$)$ \\
\hline $\mathrm{FEV}_{1} / \mathrm{FVC}<\mathrm{LLN}$ & $9(20.9 \%)$ \\
\hline $\mathrm{FEV}_{1} / \mathrm{FVC}<0.70$ & 5 (I $1.6 \%)$ \\
\hline
\end{tabular}

Abbreviations: SD, standard deviation; FEVI, forced vital capacity in I sec; FVC, forced vital capacity; \%pred., percentage of predicted value; L, litres; AFO, air flow obstruction; LLN, lower limit of normal.

6 months and 18months (Visit 5), there were additional significant increases in $\mathrm{FEV}_{1}$ (median change $=3.5 \%$ pred., $\mathrm{P}<0.01$ ), $\mathrm{FVC}$ (median change $=3.5 \%$ pred., $\mathrm{P}=0.07$ ) and a non-significant increase in $\mathrm{FEV}_{1} / \mathrm{FVC}(\mathrm{P}=0.25)$.

On plethysmography (Figure 2), there was a small but significant increase in TLC and FRC between 2 months and 6 months (median change $3.5 \%$ of pred. $(\mathrm{P}=0.02)$; and median change $2.3 \%$ of pred. $(\mathrm{P}=0.05)$, respectively), but nonsignificant increases in $\mathrm{RV}(\mathrm{P}=0.35)$. Between 6 and 18 months (ie, after treatment completion), a statistically significant increase in TLC (median change $5.9 \%$ pred., $\mathrm{P}<0.01$ ), RV (median change $19.4 \%$ of pred., $\mathrm{P}<0.01$ ) and FRC (median change $9.3 \%$ pred. $\mathrm{P}<0.01$ ) was observed. There was no significant change in IC between 2 and 6 months, or between 6 and 18 months ( $\mathrm{P}=0.55$ and $\mathrm{P}=0.41$, respectively) (see Table 2 ).

The DLco (\%pred.) did not change significantly between 2 and 6 months $(\mathrm{P}=0.18)$, or between 6 and 18 months $(\mathrm{P}=0.07)$.
The six-minute walk distance did not improve significantly at two months (median change $15 \mathrm{~m}, \mathrm{P}=0.06$ ), or thereafter. A statistically significant but not clinically important decrease (improvement) in SGRQ total score was observed between 6 and 18 months ( -1.0 units, $\mathrm{P}<0.01$ ).

One year after completion of treatment (ie, Visit 5), seven patients $(16.3 \%)$ had AFO, and in six (14.0\%) $\mathrm{FEV}_{1}$ $/$ FVC was $<0.70$. Gas trapping was found in $14(32.6 \%)$, and restriction in eight $(18.6 \%)$ patients. DLco was reduced in 33 of 42 patients $(78.6 \%)$, and DLco/VA was reduced in 14 of 42 patients (33.3\%). The combinations of $\mathrm{AFO}$, restriction, gas trapping and reduced DLco are shown in Figure 3.

\section{Changes in Quantitative Imaging Over Time (Table 3)}

On CT imaging the TLC increased significantly between 2 and 6 months (median change $0.22 \mathrm{~L}, \mathrm{P}=0.01$ ), but not between 6 and 18 months $(\mathrm{P}=0.99)$.

The median gas-trapping scores (ie, $<-856 \mathrm{HU}$ on RV scan) measured at 2 and 6 months were not significantly different $(\mathrm{P}=0.19)$, but showed small but significant increases between 6 and 18 months $(\mathrm{P}=0.04)$. The median difference in emphysema score (ie, LAA $<-950 \mathrm{HU}$ ) between 2 and 6 months and between 6 and 18 months were non-significant $(0.92 \%, \mathrm{P}=0.20$ and $0.90 \%, \mathrm{P}=0.11$, respectively). The median fibrosis score decreased significantly between 2 and 6 months $(\mathrm{P}<0.01)$, but changes between 6 and 18 months were non-significant $(\mathrm{P}=0.07)$.

At 18 months, 14 of 19 (73.7\%) patients had $>5 \%$ GTS on expiratory CT scan, seven of whom $(36.8 \%)$ had a GTS value $>10 \%$, and two $(10.5 \%)$ GTS $>20 \%$. Fibrosis scores, and radiological volumes are reported in Table 3.

\section{Correlation Between Physiologic and Radiologic Measures Over Time}

The overall change in RV/TLC (measured by plethysmography) between 2 and 18 months correlated with changes in radiological GTS $\left(\mathrm{R}^{2}=0.23, \mathrm{P}=0.04\right)$. This correlation was observed only after 6 months $\left(\mathrm{R}^{2}=0.36, \mathrm{P}=0.01\right)$, and not at earlier time points $(\mathrm{P}=0.90)$. By contrast, the correlation between change in RV/TLC (plethysmography) and change in LAA between 2 and 18 months was not significant $\left(\mathrm{R}^{2}=0.19, \mathrm{P}=0.052\right)$. The change in $\mathrm{FEV}_{1} / \mathrm{FVC}$ between 2 and 18 months correlated poorly with change in radiological GTS $\left(\mathrm{R}^{2}=0.10, \mathrm{P}=0.18\right)$, and with change in LAA (emphysema score; $\mathrm{P}=0.11$ ). 
Table 2 Lung Physiology Data at 2, 6 (Treatment Completion) and 18 Months (One Year After Treatment Completion)

\begin{tabular}{|c|c|c|c|c|c|c|c|c|}
\hline \multirow[t]{2}{*}{$n=43$} & \multicolumn{2}{|l|}{ Baseline } & \multicolumn{2}{|c|}{2 Months } & \multicolumn{2}{|c|}{6 Months } & \multicolumn{2}{|c|}{18 Months } \\
\hline & Median & (IQR) & Median & (IQR) & Median & (IQR) & Median & (IQR) \\
\hline $\mathrm{FEV}_{\mathrm{I}}(\mathrm{L})$ & 1.97 & $(1.50-2.76)$ & 2.27 & $(1.68-2.89)$ & 2.39 & $(1.80-2.92)$ & 2.52 & (1.95-3.04) \\
\hline FEV (\%pred.) & 0.59 & $(0.50-0.77)$ & 0.73 & $(0.55-0.86)$ & 0.75 & $(0.59-0.87)$ & 0.79 & $(0.64-0.89)$ \\
\hline $\mathrm{FVC} \mathrm{(L)}$ & 2.49 & $(1.90-3.02)$ & 2.82 & $(2.22-3.44)$ & 2.88 & $(2.30-3.54)$ & 3.05 & (2.54-3.59) \\
\hline FVC (\%pred.) & 0.63 & $(0.54-0.73)$ & 0.74 & $(0.57-0.84)$ & 0.77 & $(0.62-0.87)$ & 0.81 & $(0.66-0.88)$ \\
\hline $\mathrm{FEV}_{\text {//FVC (\%) }}$ & 81.9 & $(75.2-88.1)$ & 81.6 & $(76.2-89.0)$ & 81.5 & (75.3-88.2) & 87.6 & (75.4-89.9) \\
\hline TLC (\%pred.) & - & - & 85.84 & $(72.40-93.76)$ & 86.76 & $(78.17-100.12)$ & 97.97 & $(83.96-110.69)$ \\
\hline IC (\%pred.) & - & - & 82.05 & $(68.78-97.72)$ & 83.67 & $(64.01-106.23)$ & 88.16 & $(70.00-I \mid 2.2 I)$ \\
\hline FRC (\%pred.) & - & - & 89.53 & $(67.39-106.47)$ & 97.15 & (78.63-I I6.88) & 105.43 & $(86.00-128.60)$ \\
\hline RV (\%pred.) & - & - & 132.62 & $(97.73-156.62)$ & 132.78 & $(102.01-164.17)$ & 158.29 & $(111.66-210.65)$ \\
\hline RV/TLC (\%) & - & - & 38.3 & $(32.5-46.8)$ & 37.2 & $(32.5-47.9)$ & 40.9 & $(35.4-50.0)$ \\
\hline IC/TLC ratio & - & - & 46.9 & (4I.3-53.3) & 45.4 & $(35.1-50.8)$ & 42.5 & $(36.7-46.5)$ \\
\hline DLco (\%pred.) $)^{\mathrm{a}}$ & - & - & 66.98 & $(51.42-76.46)$ & 61.68 & (52.29-73.48) & 65.31 & (52.33-77.05) \\
\hline DLco/VA (\%pred.) & - & - & 92.13 & $(83.73-103.02)$ & 89.44 & $(80.32-101.20)$ & 90.99 & $(75.67-100.35)$ \\
\hline Six-minute walk distance $(\mathrm{m})$ & 510 & $(465-545)$ & 510 & $(480-555)$ & 529 & $(480-570)$ & 520 & $(480-600)$ \\
\hline \multicolumn{9}{|l|}{ SGRQ - Total Score } \\
\hline Mean (SD) & - & - & - & - & 6.7 & $(I I .0)$ & 2.9 & (9.9) \\
\hline Median (IQR) & - & - & - & - & 1.0 & $(0.00-12.3)$ & 0.00 & $(0.00-0.4)$ \\
\hline \multicolumn{9}{|l|}{ SGRQ - Activity Score } \\
\hline Mean (SD) & - & - & - & - & 14.0 & $(25.9)$ & 3.5 & $(I 5.5)$ \\
\hline Median (IQR) & - & - & & & 0.0 & $(0.0-24.4)$ & 0.0 & $(0.0-0.0)$ \\
\hline
\end{tabular}

Notes: Plethysmography and diffusing capacity were not performed prior to 2 months due to risk to staff. St. George's Respiratory Questionnaire (SGRQ) was only performed from 6 months, as it is only validated for chronic symptoms. ${ }^{a}$ For diffusing capacity measurements, $n=42$.

Abbreviations: IQR, interquartile range; SD, standard deviation; FEVI, forced vital capacity in I sec; FVC, forced vital capacity; TLC, total lung capacity; IC, inspiratory capacity; FRC, functional residual capacity; RV, residual volume; DLco, diffusing capacity for carbon monoxide; VA, alveolar volume; L, liters; \%pred., percentage of predicted; m, meters; SGRQ, St. George's Respiratory Questionnaire.

As for RV/TLC, change in RV measured by plethysmography correlated significantly with changes in radiological GTS $\left(\mathrm{R}^{2}=0.31, \mathrm{P}=0.01\right)$, and this was similarly attributable to changes occurring after 6 months $\left(\mathrm{R}^{2}=0.43, \mathrm{P}<0.01\right)$, and not before $(\mathrm{P}=0.99)$

There was no correlation between the change in QLF $(\mathrm{mL})$ from 2 to 18 months, and any of the following plethysmography measures: change in TLC \%pred. $(\mathrm{P}=0.91)$, change in $\mathrm{RV} / \mathrm{TLC}$ ratio $(\mathrm{P}=0.17)$ or change in $\mathrm{IC} / \mathrm{TLC}(\mathrm{P}=0.24)$.

\section{Correlation of Physiology and Radiological Measures at 18 Months}

At 18 months, the $\mathrm{FEV}_{1} / \mathrm{FVC}$ correlated with radiological GTS ( $22=0.37, \mathrm{P}=0.01)$ but not with LAA (emphysema score: $\mathrm{R} 2=0.17, \mathrm{P}=0.07)$. There was also a correlation between RV/TLC measured by plethysmography and GTS on $\mathrm{CT}$ scan $(\mathrm{R} 2=0.28, \mathrm{P}=0.02)$, but not LAA $(\mathrm{R} 2=0.001, \mathrm{P}=0.89)$.
The TLC values obtained by plethysmography and CT scan correlated well $(\mathrm{R} 2=0.55, \mathrm{P}<0.01)$ at 18 months. However, the correlation between RV/TLC measured by plethysmography and by CT scan did not correlate significantly $(\mathrm{P}=0.12)$. This was primarily due to the poorer correlation between the measurement of RV with the two techniques $\left(\mathrm{R}^{2}=0.22, \mathrm{P}=0.04\right)$, and is likely explained by the difference in body position between plethysmography and CT scans (ie, erect vs supine).

There was no correlation between fibrosis scores (QLF $(\mathrm{mL}))$ and either the TLC or the IC/TLC $(\mathrm{P}=0.12$ and $\mathrm{P}=$ 0.93 , respectively).

\section{Discussion}

The results of this 18-month prospective study of young, immune-competent patients experiencing their first episode of pulmonary tuberculosis provides several insights into the pathogenesis of lung function impairment and the development of chronic lung disease during and following treatment. Firstly, at one year after completing tuberculosis 


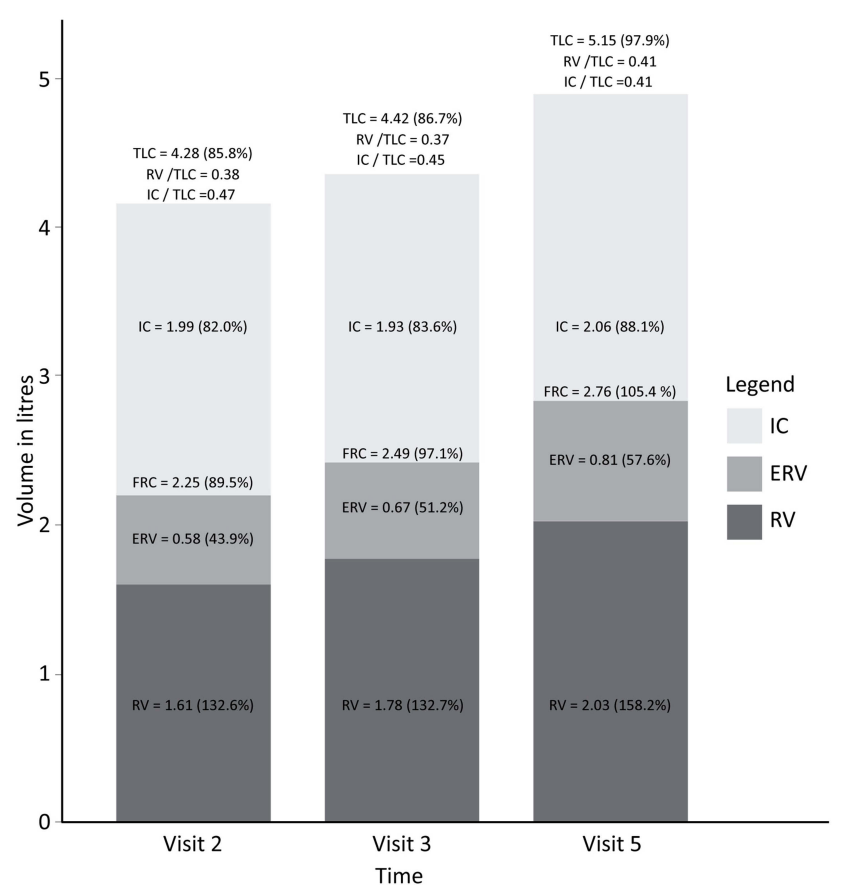

Figure 2 Median lung volumes measured by plethysmography at 2 (Visit 2), 6 (Visit 3, treatment completion) and 18 months (Visit 5).

Abbreviations: TLC, total lung capacity; IC, inspiratory capacity; FRC, functional residual capacity; RV, residual volume.

treatment three quarters of patients had at least one type of lung abnormality as defined in this study. The most common abnormality at 18 months (in 33 of 42 patients; $78.6 \%$ of the cohort) was reduced diffusing capacity for carbon monoxide. This was most commonly associated with evidence of gas trapping (in 14 of 33 patients), confirmed on quantitative CT. Seven patients (16.3\%) also had evidence of chronic airflow obstruction $\left(\mathrm{FEV}_{1} / \mathrm{FVC}\right.$ below the LLN). Restriction was detected in 8 subjects with

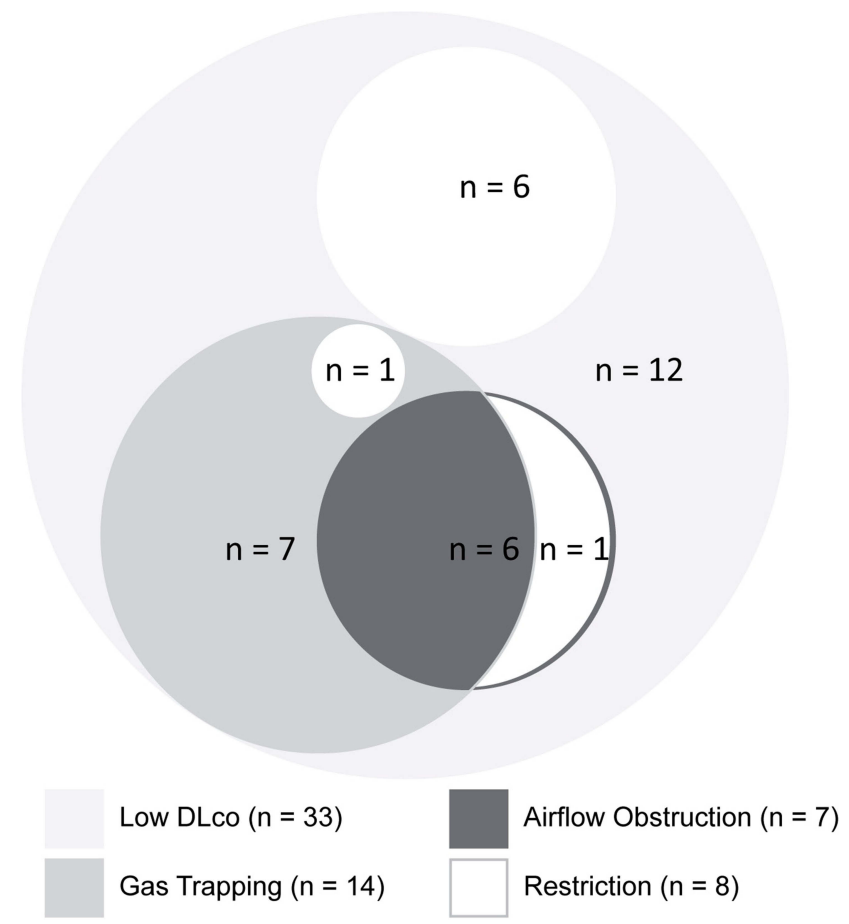

No Abnormality $(n=10)$

Figure 3 Venn diagram presenting in approximate proportions the number of patients with single and combinations of lung function abnormalities one year after completion of TB treatment (Visit 5): Airflow obstruction, gas trapping, restriction, and low diffusing capacity. Airflow obstruction was defined using the $\mathrm{FEV}_{\mathrm{I}} / \mathrm{FVC}<$ lower limit of normal definition; restriction was defined as total lung capacity (TLC) less than $80 \%$ predicted; gas trapping on plethysmography was defined as a residual volume (RV) to TLC ratio (RV/TLC) of greater than $45 \%$; and a low DLco (corrected for haemoglobin) was defined as values of less than $80 \%$ predicted.

reduced DLco, most of whom had no evidence of gas trapping or airflow obstruction.

Secondly, the changes in lung volumes progressed in the year after completion of anti-tuberculous treatment,

Table 3 Quantitative Computed Tomography Values for Subjects with Scans Performed at 2, 6 (Treatment Completion) and 18 Months (One Year After Treatment Completion)

\begin{tabular}{|c|c|c|c|c|c|c|c|c|c|}
\hline \multirow[t]{2}{*}{$n=\mathbf{2 0}$} & \multicolumn{2}{|c|}{2 Months } & \multicolumn{2}{|c|}{6 Months } & \multicolumn{2}{|c|}{18 Months } & \multirow{2}{*}{$\begin{array}{l}\text { Change } 2 \\
\text { to } 6 \\
\text { Months } \\
\text { P-value }\end{array}$} & \multirow{2}{*}{$\begin{array}{l}\text { Change } 6 \\
\text { to } 18 \\
\text { Months } \\
\text { P-value }\end{array}$} & \multirow{2}{*}{$\begin{array}{l}\text { Change } 2 \\
\text { to I8 } \\
\text { Months }\end{array}$} \\
\hline & Median & (IQR) & Median & (IQR) & Median & (IQR) & & & \\
\hline TLC (L) & 3.91 & $(3.14-4.44)$ & 4.01 & (3.39-4.57) & 4.06 & $(3.46-4.69)$ & 0.01 & 0.99 & 0.16 \\
\hline $\mathrm{RV}(\mathrm{L})$ & 1.89 & $(1.58-2.36)$ & 1.79 & $(1.56-2.19)$ & $1.96^{\mathrm{a}}$ & $(1.65-2.19)$ & 0.41 & 0.86 & 0.77 \\
\hline LAA $\left(\%^{\mathrm{b}}\right)$ & $9.07 \%$ & $(4.57-11.04)$ & $8.76 \%$ & $(6.78-11.63)$ & $9.84 \%$ & $(7.75-14.45)$ & 0.20 & 0.11 & 0.02 \\
\hline GTS (\%) & $5.26 \%$ & $(2.79-9.16)$ & $6.85 \%$ & $(4.23-10.98)$ & $7.13 \%^{\mathrm{a}}$ & $(4.79-15.87)$ & 0.19 & 0.04 & 0.02 \\
\hline QLF (\%) & $2.15 \%$ & $(I .3 I-3.4 I)$ & $1.14 \%$ & $(0.72-1.67)$ & $0.76 \%$ & $(0.57-1.55)$ & $<0.01$ & 0.11 & $<0.01$ \\
\hline QLF (mL) & 84.67 & $(63.50-139.12)$ & 38.30 & $(3 I .5 I-69.5 I)$ & 30.48 & $(21.85-72.13)$ & $<0.01$ & 0.07 & $<0.01$ \\
\hline
\end{tabular}

Notes: ${ }^{a} \mathrm{n}=19$. ${ }^{b}$ Percentage of total lung volume. ${ }^{c} \mathrm{P}$-value for paired Wilcoxon test.

Abbreviations: TLC, total lung capacity; RV, residual volume; LAA, low-attenuation areas on inspiratory scan (ie, <-950HU, emphysema score); GTS, gas-trapping score on expiratory scan (ie, <-856HU); QLF, quantity of lung fibrosis; L, litres; mL, milliliters. 
presumably as a consequence of gas trapping rather than emphysema. The increase in TLC one year after treatment completion was largely due to a large increase in RV that was not associated with an increase in IC. The CT imaging confirmed that this increase in RV/TLC ratio correlated more closely with radiological gas-trapping scores, than with either emphysema or fibrosis scores. No correlation was found between the fibrosis scores and any physiological parameter, including TLC and IC/TLC. Together, these findings suggest that the physiological gas trapping is independent of emphysema, and may point to developing airflow limitation at the level of small airways. This hypothesis is supported by lung images in individual patients showing gas trapping on expiratory lung scans one year after treatment, in spite of good resolution of tuberculous pulmonary infiltrates (Figure 4). This explanation is plausible given the extensive bronchiolar involvement seen in more than $90 \%$ of patients with active tuberculosis disease. ${ }^{11}$ The mechanisms responsible for the consistently observed finding of reduced $\mathrm{DL}_{\mathrm{CO}}$ is less clear, but potential involvement of vasculature within the bronchovascular bundle in and around small airways is one potential mechanism. ${ }^{15}$
Several large multi-centre studies and systematic reviews have demonstrated the association of previous tuberculosis and spirometric abnormalities. ${ }^{3-6,13,14,19,20}$ However, these and several smaller studies have been performed without the use of plethysmography or quantitative imaging. In this present study, we found spirometry to be relatively insensitive in identifying post-tuberculosis physiological abnormalities; almost twice as many patients had plethysmographic evidence of gas trapping as compared with spirometry, and almost $80 \%$ had a reduction in diffusing capacity.

Our study has a number of strengths, these include its prospective nature and the enrollment of a young population without previous episodes of PTB, and without known chronic respiratory or cardiac diseases, or recognized impaired immune-incompetency. All PTB infections were drug-sensitive and only those who completed treatment and follow-up were included in analysis. Smoking although not an exclusion criterion, was light (median 3.0 pack-years in those who smoked). It is thus reasonable to assume, that despite the small sample size, the majority of findings are likely primarily attributable to the chronic consequence of tuberculosis.

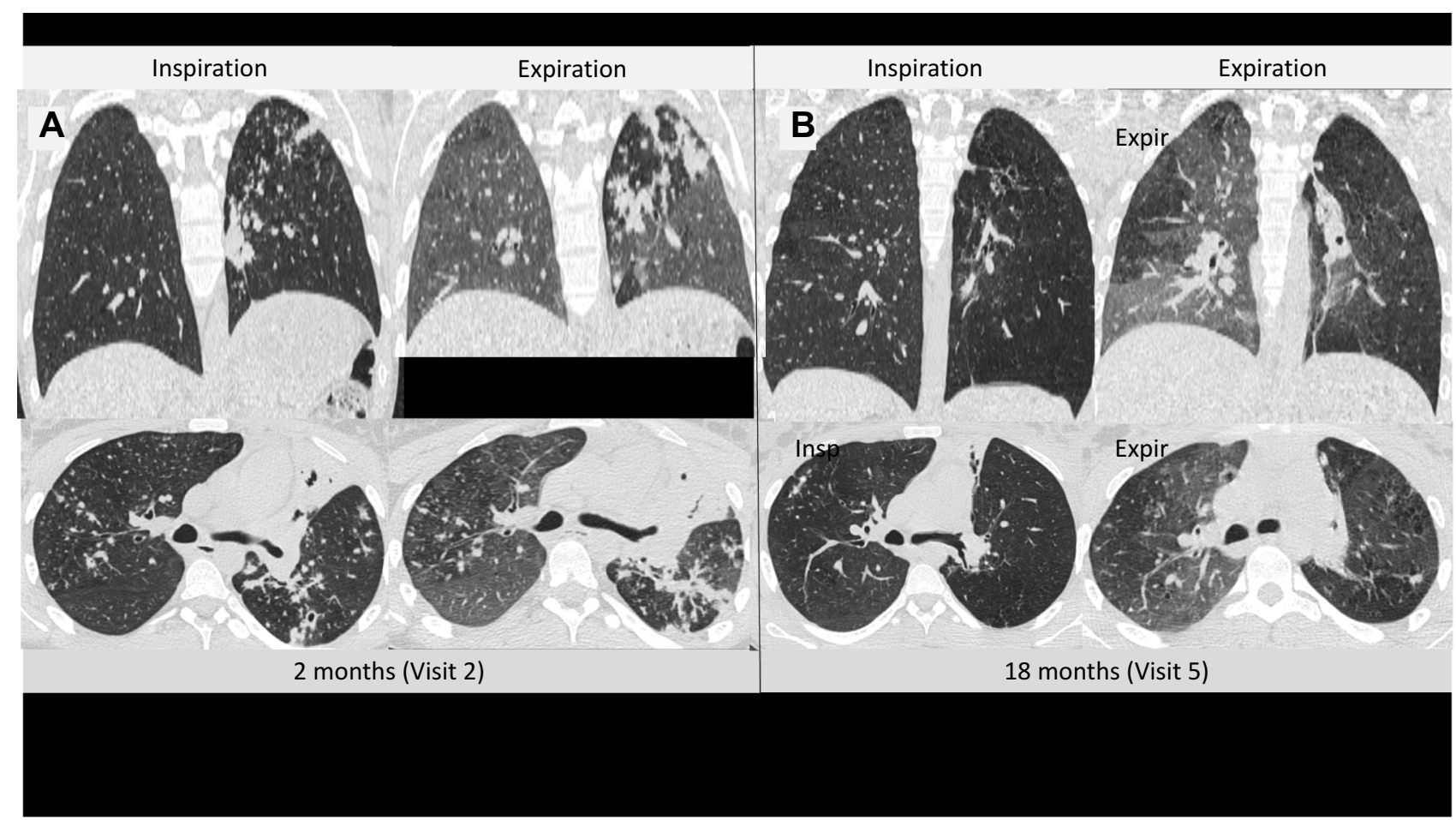

Figure 4 Inspiratory and expiratory, axial and coronal computed tomography images for a 20-year-old female, after 2 months of tuberculosis treatment (Visit 2) (A), and I year after treatment completion (Visit 5) (B). Resolution of infiltrates of tuberculosis and improvement in inspiratory lung volumes was found. However, areas of gas trapping can be seen on the Visit 5 expiratory scan which were not present at 2 months, and are particularly prominent in regions where infiltrates of tuberculosis have resolved. 
However, there are also a number of weaknesses, which include its small size with the potential for selection bias, loss of six patients in follow-up, and significant but small correlations. The size of the study may have been underpowered to assess smaller effect of potential cofactors (eg, smoking). Further, in order to limit radiation exposures, timepoints for $\mathrm{CT}$ imaging were varied to capture different stages of treatment and follow-up. Finally, owing to potential infection risk, diffusing capacity and body plethysmography were not performed at the baseline visit. However, in spite of these limitations, statistically significant trends were observed.

\section{Conclusions}

This young, previously healthy, adult population, demonstrated a significant burden of airflow obstruction (one in six) and residual chronic changes one year after the first episode of treated tuberculosis. The residual disease burden was underestimated by spirometry. Gas trapping (in one third) and reduced DLco (in three quarters) were more common than restriction (one in five). The RV and RV/ TLC continued to increase in the year after treatment completion and correlated best with radiological gas trapping. This is consistent with the hypothesis of residual and evolving obstructive changes in the small airways after tuberculosis.

\section{Abbreviations}

AFO, airflow obstruction; CT, computed tomography; DLco, diffusing capacity for carbon monoxide; $\mathrm{FEV}_{1}$, forced expiratory volume in $1 \mathrm{sec}$; FVC, forced vital capacity; GLI, Global Lung Initiative; GTS, gas-trapping score; HAA, high-attenuation areas; HRCT, high-resolution computed tomography; IC, inspiratory capacity; L, liters; LAA, low-attenuation areas; LLN, limit of normal; PTB, pulmonary tuberculosis; pMDI, pressurized metered dose inhaler; QLF, quantity of lung fibrosis; QLF (\%), quantity of lung fibrosis (percentage); QLF (mL), quantity of lung fibrosis (millilitre volume); RV, residual volume; SGRQ, St. George's Respiratory Questionnaire; TLC, total lung capacity; $\mathrm{V}_{\mathrm{A}}$, alveolar volume.

\section{Notification of Prior Abstract Publication/Presentation}

This data has not been published elsewhere but was presented as abstract for the European Respiratory Society Meeting, September 2019.

\section{Acknowledgments}

Role of sponsors:

Funders did not have any role in the study design, data collection, data analysis, manuscript preparation or decision to publish.

Other contributions:

We would like to acknowledge the technical assistance of Bronwyn Hendricks, Helena Olckers, Nolubabalu Bam and La-Donna Kappa, at the University of Cape Town Lung Institute.

\section{Author Contributions}

Protocol design: BA, CC, JG, RvZS, EB, RD; Data Collection: BA, RvZS, RD; Analysis: BA, EM, GK, JG, CC, EB; Manuscript Preparation: BA, EM, GK, CC, JG, RvZS, EB, RD. All authors contributed to data analysis, drafting or revising the article, gave final approval of the version to be published, and agree to be accountable for all aspects of the work.

\section{Funding}

Funding received from the University of Cape Town Lung Institute for funding the radiology component and Global Alliance for TB Drug Development for access to their biostorage cohort. E.M. is funded by a Strategic Health Innovation Partnership (SHIP) grant from the South African (SA) Department of Science and Technology (DST) and SA Medical Research Council (SAMRC) to the South African Tuberculosis Bioinformatics Initiative (SATBBI), and E.B. received funding from the National Research Foundation (NRF).

\section{Disclosure}

Dr Brian Allwood reports speaker honoraria from Novartis, outside the submitted work. Professor Kim reports personal fee from MedQIA outside the submitted work.

Professor Richard van Zyl-Smit reports personal fees from AstraZeneca CIPLA, Roche, Novartis, Pfizer, ASPEN/GSK and MSD outside the submitted work.

Dr. Cooper reports grants from NIH/NHLBI, Foundation NIH and COPD Foundation, during the conduct of the study; personal fees from PulmonX, GlaxoSmithKline, NUVAIRA and MGC Diagnostics, outside the submitted work.

Professor Eric Bateman reports personal fees from ALK, AstraZeneca, Boehringer Ingelheim, Menarini, 
Novartis, Orion, Regeneron and Sanofi outside the submitted work.

The authors report no other conflicts of interset in this work.

\section{References}

1. Harries AD, Ade S, Burney P, Hoa NB, Schluger NW, Castro JL. Successfully treated but not fit for purpose: paying attention to chronic lung impairment after TB treatment. Int J Tuberc Lung Dis. 2016;20 (8):1010-1014. doi:10.5588/ijtld.16.0277

2. O'Toole RF, Shukla SD, Walters EH. TB meets COPD: an emerging global co-morbidity in human lung disease. Tuberculosis. 2015;95 (6):659-663. doi:10.1016/j.tube.2015.08.005

3. Amaral AFS, Coton S, Kato B, et al. Tuberculosis associates with both airflow obstruction and low lung function: BOLD results. Eur Respir J. 2015;46(4):1104-1112. doi:10.1183/13993003.02325-2014

4. Menezes AMB, Hallal PC, Perez-Padilla R, et al. Tuberculosis and airflow obstruction: evidence from the PLATINO study in Latin America. Eur Respir J. 2007;30(6):1180-1185. doi:10.1183/09031936.00083507

5. Caballero A, Torres-Duque CA, Jaramillo C, et al. Prevalence of COPD in five colombian cities situated at low, medium, and high altitude (PREPOCOL study). Chest. 2008;133(2):343-349. doi:10.1378/chest.07-1361

6. Lam KB, Jiang CQ, Jordan RE, et al. Prior TB, smoking, and airflow obstruction: a cross-sectional analysis of the guangzhou biobank cohort study. Chest. 2010;137(3):593-600. doi:10.1378/chest.09-1435

7. Ko Y, Lee YM, Lee HY, et al. Changes in lung function according to disease extent before and after pulmonary tuberculosis. Int $J$ Tuberc Lung Dis. 2015;19(5):589-595. doi:10.5588/ijtld.14.0454

8. Muñoz-Torrico M, Rendon A, Centis R, et al. Is there a rationale for pulmonary rehabilitation following successful chemotherapy for tuberculosis? J Bras Pneumol. 2016;42(5):374-385. doi:10.1590/ S1806-37562016000000226

9. Pasipanodya JG, Miller TL, Vecino M, et al. Pulmonary impairment after tuberculosis. Chest. 2007;131(6):1817-1824. doi:10.1378/ chest.06-2949
10. Plit ML, Anderson R, Van Rensburg CEJ, et al. Influence of antimicrobial chemotherapy on spirometric parameters and pro-inflammatory indices in severe pulmonary tuberculosis. Eur Respir J. 1998;12(2):351-356. doi:10.1183/09031936.98.12020351

11. Long R, Maycher B, Dhar A, Manfreda J, Hershfield E, Anthonisen N. Pulmonary tuberculosis treated with directly observed therapy serial changes in lung structure and function. Chest. 1998;113 (4):933. doi:10.1378/chest.113.4.933

12. Vecino M, Pasipanodya JG, Slocum P, et al. Evidence for chronic lung impairment in patients treated for pulmonary tuberculosis. $J$ Infect Public Health. 2011;4(5-6):244-252. doi:10.1016/j. jiph.2011.08.005

13. Ross J, Ehrlich RI, Hnizdo E, White N, Churchyard GJ. Excess lung function decline in gold miners following pulmonary tuberculosis. Thorax. 2010;65(11):1010-1015. doi:10.1136/thx.2009.129999

14. Byrne AL, Marais BJ, Mitnick CD, Lecca L, Marks GB. Tuberculosis and chronic respiratory disease: a systematic review. Int $J$ Infect Dis. 2015;32:138-146. doi:10.1016/j.jijid.2014.12.016

15. Allwood BW, Gillespie R, Galperin-Aizenberg M, et al. Obstructive pulmonary disease in patients with previous tuberculosis: pathophysiology of a community-based cohort. South Afr Med J. 2017;107 (5):440. doi:10.7196/SAMJ.2017.v107i5.12118

16. Pellegrino R, Viegi G, Brusasco V, et al. Interpretative strategies for lung function tests. Eur Respir J. 2005;26(5):948-968. doi:10.1183/ 09031936.05.00035205

17. Global Initiative for Chronic Obstructive Lung Disease. Global Strategy for Diagnosis, Management, and Prevention of COPD. Available from: https://goldcopd.org/gold-reports/. Accessed February 15, 2020.

18. Kim HG, Tashkin DP, Clements PJ, et al. A computer-aided diagnosis system for quantitative scoring of extent of lung fibrosis in scleroderma patients. Clin Exp Rheumatol. 2010;28(5 Suppl 62):S26-S35.

19. Allwood BW, Myer L, Bateman ED. A systematic review of the association between pulmonary tuberculosis and the development of chronic airflow obstruction in adults. Respiration. 2013;86(1):76-85. doi: $10.1159 / 000350917$

20. Willcox PA, Ferguson AD. Chronic obstructive airways disease following treated pulmonary tuberculosis. Respir Med. 1989;83 (3):195-198. doi:10.1016/S0954-6111(89)80031-9

International Journal of Chronic Obstructive Pulmonary Disease

Dovepress

\section{Publish your work in this journal}

The International Journal of COPD is an international, peer-reviewed journal of therapeutics and pharmacology focusing on concise rapid reporting of clinical studies and reviews in COPD. Special focus is given to the pathophysiological processes underlying the disease, intervention programs, patient focused education, and self management protocols. This journal is indexed on PubMed Central, MedLine and CAS. The manuscript management system is completely online and includes a very quick and fair peer-review system, which is all easy to use. Visit http://www.dovepress.com/testimonials.php to read real quotes from published authors. 